\title{
Ustekinumab Treatment of Erythrodermic Psoriasis Occurring after Physical Stress: A Report of Two Cases
}

\author{
Rosita Saraceno $^{\mathrm{a}}$ Marina Talamonti $^{\mathrm{a}}$ Marco Galluzzo $^{\mathrm{a}}$ \\ Andrea Chiricozzi ${ }^{\mathrm{a}}$, Antonio Costanzo ${ }^{\mathrm{a}}$ Sergio Chimenti ${ }^{\mathrm{a}}$ \\ a'Departments of Dermatology and Internal Medicine, University of Rome 'Tor Vergata', \\ Rome, Italy
}

\section{Key Words}

Ustekinumab · Erythrodermic psoriasis · Anti-TNFa

\begin{abstract}
Erythrodermic psoriasis (EP) is a severe form of psoriasis precipitated by numerous factors, including physical stress, infections, and drugs. The disease represents a therapeutic challenge, and little is known about its response to ustekinumab. Though the efficacy of ustekinumab has been extensively studied in chronic plaque psoriasis, no trials have been carried out in EP. We report the case of 2 patients, 1 male and 1 female, who showed EP despite being treated with etanercept and methotrexate for chronic plaque psoriasis, respectively. The patients were treated with ustekinumab at a dosage of $45 \mathrm{mg}$ s.c. They showed a significant improvement in their Psoriasis Area and Severity Index score after only 4 weeks of ustekinumab therapy, and further improvements were observed throughout the treatment. In our experience, ustekinumab has been proven safe and effective, without increasing the dosage, in controlling and preventing the occurrence of erythrodermic flares. Ustekinumab therapy may therefore be considered a valid therapeutic option for the treatment of EP, even in cases where other biological agents have failed.
\end{abstract}




\section{Introduction}

Erythrodermic psoriasis (EP) is a severe variant of psoriasis with a reported prevalence among psoriatic patients ranging from 1.5 to $31 \%$. Most commonly, this variant may be a manifestation of unstable chronic plaque-type psoriasis, precipitated by infection, drugs, or a sudden withdrawal of corticosteroids. Left untreated, it may lead to serious morbidity and even mortality. Clinically, it is characterized by diffuse red-violet erythema and fine scaling involving all or almost all of the body surface area. Erythrodermic flare is commonly associated with chills, exudation, edema, onycholysis, onychodystrophy, weight loss, pruritus, and fever [1-3]. This condition is often difficult to manage, and the therapies that are currently available may prove to be unsatisfactory. Biological agents offer a new alternative therapeutic approach [4], though there are no controlled trials to support their use, particularly as a long-term therapy option.

Ustekinumab is a human monoclonal antibody that, binding to the shared p40 protein subunit of human interleukin (IL)-12 and -23 with high affinity and specificity, prevents interaction between both cytokines and the surface IL-12R $\beta 1$ receptor. To date, different clinical studies have shown satisfactory and rapid efficacy of ustekinumab in the treatment of chronic plaque psoriasis, with high capacity to safely control the disease, even in the longterm period [5-7].

We report 2 EP cases successfully treated with ustekinumab.

\section{Case Reports}

Case 1

A 59-year-old man with a 19-year history of moderate-to-severe plaque psoriasis was referred to our department in August 2011 due to a flare of erythroderma involving approximately $100 \%$ of his body surface area and a Psoriasis Area and Severity Index (PASI) score of 59 (fig. 1a).

He had been previously treated with topical and systemic drugs, such as cyclosporine A and methotrexate that were interrupted for high blood pressure and loss of efficacy, respectively. Without therapy, his psoriasis worsened. Considering the severity of the skin clinical features and his intolerance of conventional systemic therapies, the patient was considered as 'high need' for biological drugs and was treated with efalizumab in December 2003. The patient withdrew from efalizumab treatment in March 2004 for ineffectiveness and the onset of erythroderma. Hospitalization was necessary, and etanercept (50 mg s.c./week) was administered with a good control of the disease from April 2004 until July 2011.

In July 2011, shortly following cataract surgery on the left eye, the patient developed generalized erythroderma with fever $\left(38.5^{\circ} \mathrm{C}\right)$. The therapy with etanercept was discontinued, and the patient was treated with systemic corticosteroid therapy (prednisone 50 $\mathrm{mg}$ /day) obtaining an unsatisfactory disease control. In August 2011, severe erythroderma and fever associated with chills and pruritus (itching VAS: 90) occurred. Complete laboratory and instrumental tests were performed, including chest X-ray, ECG, QuantiFeron TB-Gold, complete blood count, complete liver profile, creatinine, auto-antibodies (ANA, anti-dsDNA, ENA, LAC, anti-cardiolipin, anti-citrullin), C-reactive protein , tumor markers, hemoculture, and urinoculture; no significant abnormal results were found. We therefore decided to start therapy with ustekinumab $45 \mathrm{mg}$ s.c. (body weight was $82 \mathrm{~kg}$ ). 
After 4 weeks of treatment, the patient showed an excellent and rapid improvement (fig. 1b) with a reduction of the PASI score to 10 and the absence of fever and itching. After 12 weeks of therapy, the patient showed complete resolution of the clinical picture with the presence of only mild scaling and a PASI score of 3 (fig. 1c). Currently, the patient has been undergoing treatment for 12 months, maintaining a PASI score of 75 with no adverse events.

Case 2

The second case we report is a 53-year-old woman suffering from plaque-type psoriasis since adolescence, developing joint involvement since the age of 40. Various topical therapies (e.g., tar, corticosteroids, vitamin D analogues) and systemic therapies, both traditional and biological, were discontinued due to the lack of efficacy or the occurrence of adverse events (e.g., renal failure, leukopenia).

Because of the worsening of joint symptoms, therapy with metrotexate was reintroduced for 3 months, but ultimately suspended because of the onset of erythroderma. Treatment with intravenous methylprednisolone and ranitidine hydrochloride temporarily improved her clinical condition, but an erythrodermic relapse (PASI score: 40) was observed despite the patient undergoing treatment with small doses of oral corticosteroids (fig. 2a).

Therapy with ustekinumab at the dosage of $45 \mathrm{mg}$ s.c. was started as monotherapy, and after only 2 weeks (fig. 2b), the patient experienced an improvement of her clinical features with a visible reduction of erythema and scaling, achieving an improvement of her PASI score from baseline exceeding 75\% (PASI score: 8.4). A second dose was administered 4 weeks later, and after 3 months, the patient showed a PASI score of 0 with a satisfactory control of articular symptoms, using ketoprofen as needed (fig. 2c). To date, the patient has been treated for 64 weeks, maintaining a PASI score of 90 with no reported side effects.

\section{Discussion}

The current management of EP is difficult, not standardized, and often unsatisfactory. Traditional systemic therapies for EP include methotrexate, cyclosporine, and oral retinoids, though systemic steroids are still used in many cases $[1,3]$.

Numerous factors limit the use of conventional therapeutic options, namely long-term organ-specific toxicity and/or the risk of opportunistic infections due to their immunosuppressive effect. While the efficacy of biological agents has been studied extensively in psoriasis vulgaris, studies regarding EP are scarce and limited to small case series. As previously reported $[8,9]$, we observed a striking and rapid response to ustekinumab in the treatment of EP. To our knowledge, this is the first report on the long-term treatment of EP using ustekinumab without any increase in dosage [10], demonstrating its ability to control and prevent the occurrence of erythrodermic flare with an excellent safety profile and a satisfactory compliance.

In conclusion, our study reveals the use of ustekinumab to be an effective therapeutic option for EP cases where conventional therapies and anti-TNF agents have failed.

\section{Disclosure Statement}

M. Talamonti, M. Galluzzo, and A. Chricozzi have no conflicts of interest. A. Costanzo is speaker for Pfizer, Janssen-Cilag, and Abbott; S. Chimenti is speaker for Pfizer, Merck Sharp \& Dohme, Janssen-Cilag, and Abbott. 
Saraceno et al.: Ustekinumab Treatment of Erythrodermic Psoriasis Occurring after Physical Stress: A Report of Two Cases

\section{References}

1 Christophers E, Mrowietz U: Psoriasis; in Freedberg IM, Eisen AZ, Wolff K, Austen KF, Goldsmith LA, Katz SI, et al: Fitzpatrick's Dermatology in General Medicine. New York, McGraw-Hill, 2003, pp 407-427.

-2 Roth PE, Grosshans E, Bergoend H: Psoriasis: development and fatal complications. Ann Dermatol Venereol 1991;118:97-105.

-3 Boyd AS, Menter A: Erythrodermic psoriasis: precipitating factors, course, and prognosis in 50 patients. J Am Acad Dermatol 1989;21:985-991.

4 Esposito M, Mazzotta A, de Felice C, Papoutsaki M, Chimenti S: Treatment of erythrodermic psoriasis with etanercept. Br J Dermatol 2006;155:156-159.

-5 Krueger GG, Langley RG, Leonardi C, Yeilding N, Guzzo C, Wang Y, Dooley LT, Lebwohl M; CNTO 1275 Psoriasis Study Group: A human interleukin-12/23 monoclonal antibody for the treatment of psoriasis. N Engl J Med 2007;356:580-592.

-6 Leonardi CL, Kimball AB, Papp K, Yeilding N, Guzzo C, Wang Y, Li S, Dooley LT, Gordon KB; PHOENIX 1 study investigators: Efficacy and safety of ustekinumab, a human interleukin-12/23 monoclonal antibody, in patients with psoriasis: 76-week results from a randomized, double-blind, placebo-controlled trial (PHOENIX 1). Lancet 2008;371:1665-1674.

-7 Papp KA, Langley RG, Lebwohl M, Krueger GG, Szapary P, Yeilding N, Guzzo C, Hsu MC, Wang Y, Li S, Dooley LT, Reich K; PHOENIX 2 study investigators: Efficacy and safety of ustekinumab, a human interleukin-12/23 monoclonal antibody, in patients with psoriasis: 52 -week results from a randomized, double-blind, placebocontrolled trial (PHOENIX 2). Lancet 2008;371:1675-1684.

8 Wang TS, Tsai TF: Clinical experience of ustekinumab in the treatment of erythrodermic psoriasis: a case series. J Dermatol 2011;38:1096-1099.

-9 Santos-Juanes J, Coto-Segura P, Mas-Vidal A, Galache Osuna C: Ustekinumab induces rapid clearing of erythrodermic psoriasis after failure of antitumour necrosis factor therapies. Br J Dermatol 2010;162:11441114.

10 Castiñeiras I, Fernández-Diaz L, Juárez Y, Lueiro M: Sustained efficacy of ustekinumab in refractory erythrodermic psoriasis after failure of antitumor necrosis factor therapies. J Dermatol 2012;39:730-731. 


\section{Case Reports in Dermatology}

a
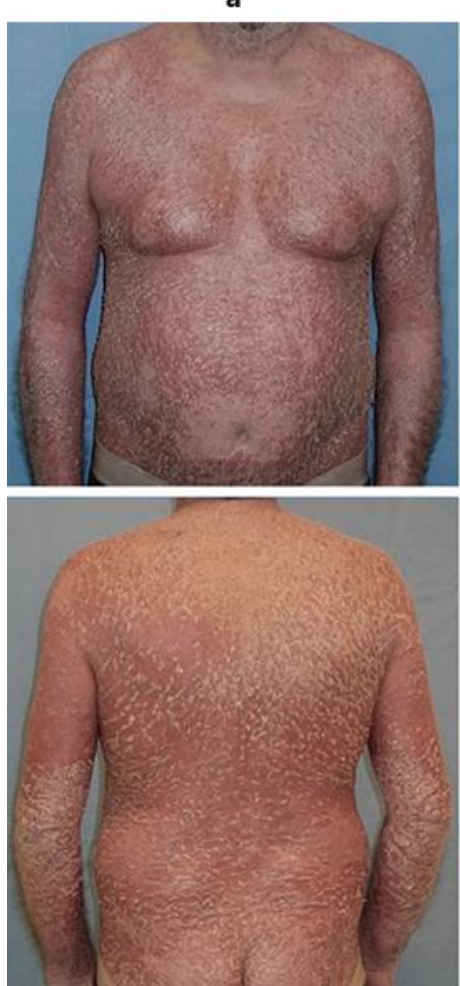

Case Rep Dermatol 2013;5:254-258

DOI: $10.1159 / 000348645$ Saraceno et al.: Ustekinumab Treatment of Erythrodermic Psoriasis Occurring afte
Physical Stress: A Report of Two Cases b
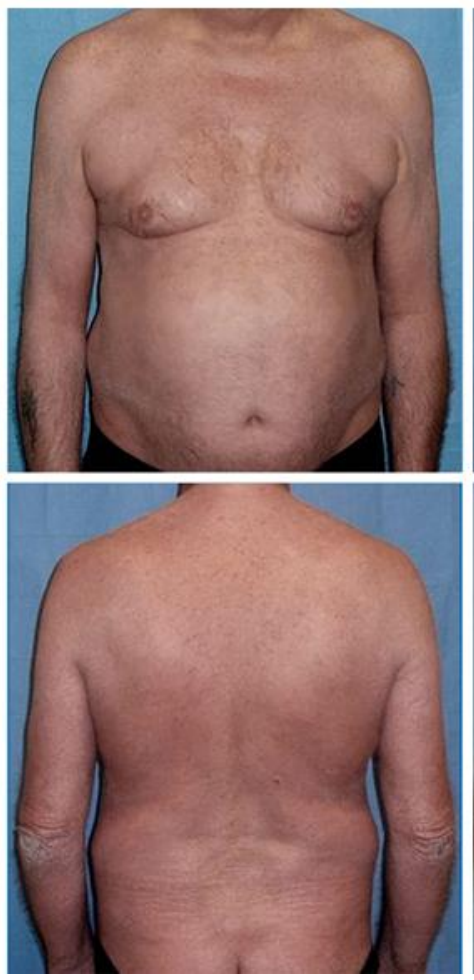

C
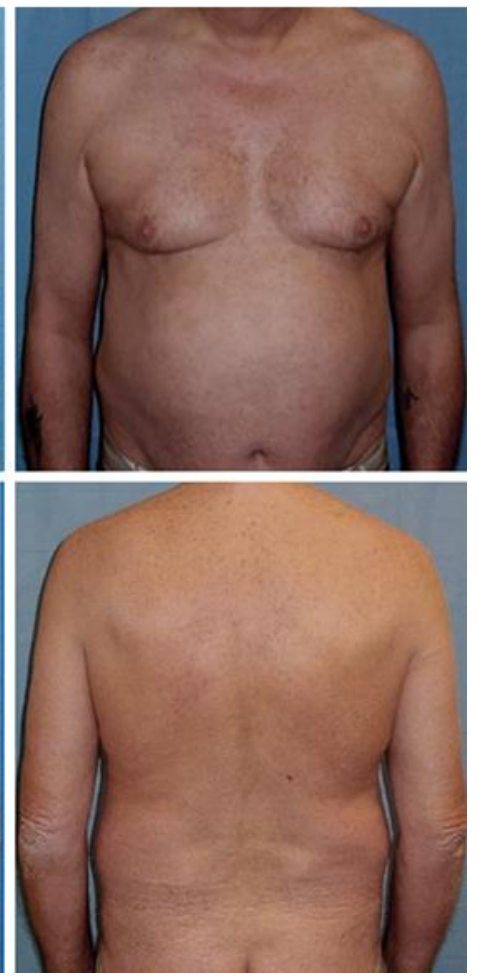

Fig. 1. First patient. a Baseline; b after 4 weeks of ustekinumab treatment; c after 12 weeks of ustekinumab treatment. 


\section{Case Reports in Dermatology}
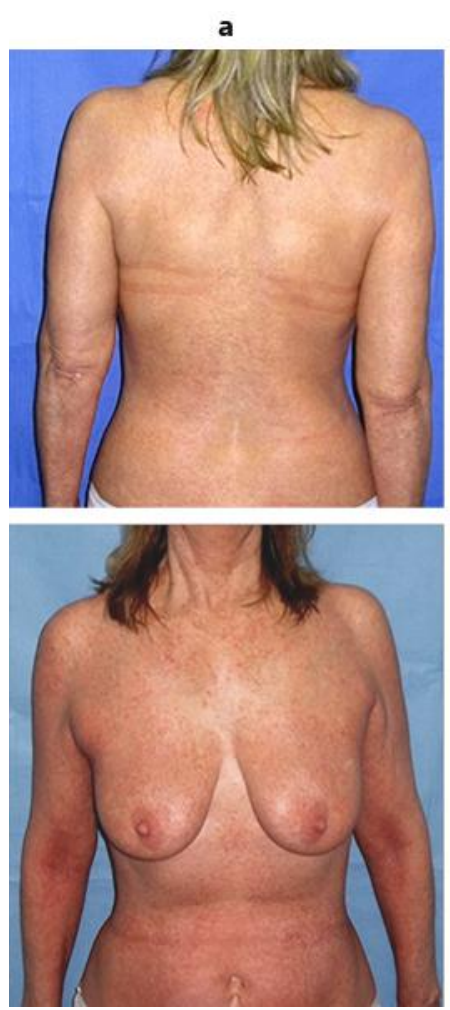

Case Rep Dermatol 2013;5:254-258

DOI: 10.1159/000348645

Saraceno et al: Ustekinumab Treatment of Erythrod

Saraceno et al.: Ustekinumab Treatment of Erythrodermic Psoriasis Occurring after Physical Stress: A Report of Two Cases
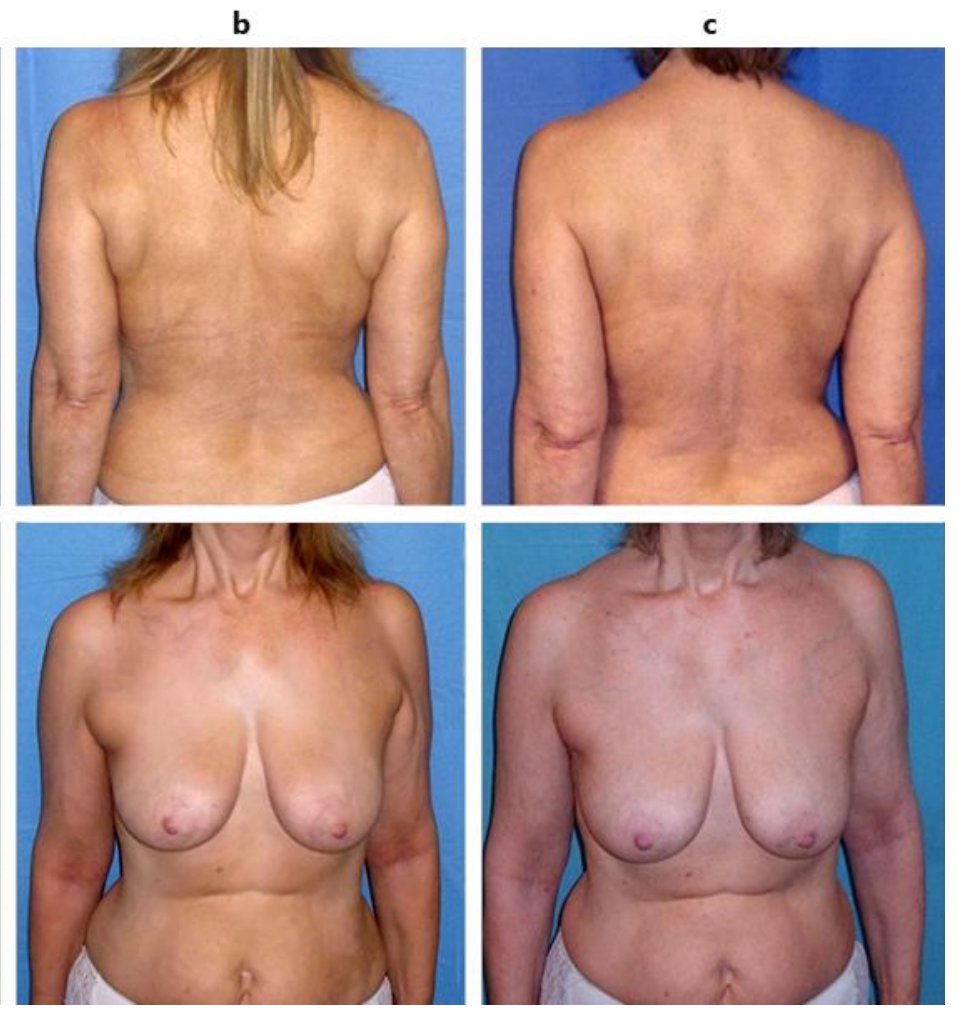

Fig. 2. Second patient. a Baseline; b after 2 weeks of ustekinumab treatment; c after 12 weeks of ustekinumab treatment. 